\title{
A risk management approach to the design of contraceptive implants
}

\author{
Sam Rowlands, Mark-Alexander Sujan, Matthew Cooke
}

\begin{abstract}
This article assesses the risks associated with the insertion and removal of contraceptive implants. Risks to patient safety relate to the way the insertion device is designed and used, rather than to the pharmacological properties of the implant itself. Risks associated with removal are not amenable to thoughtful design. A systems approach is

insertion of implants. The design of the Implanon ${ }^{\circledR}$ applicator is critically examined and suggestions made as to how it could be improved in such a way as to reduce errors in its use. The exercise undertaken has coincided with the imminent launch of the redesigned applicator of the new contraceptive implant, Nexplanon ${ }^{\circledR}$. Preliminary comments are made about the new features of Nexplanon.
\end{abstract} taken, the assumption being that human errors are symptoms of underlying systems deficiencies rather than causes of adverse events.

The insertion procedure is broken down into five key steps. Errors in these steps contribute to non-insertion and deep

\section{Introduction}

Risk management is generally understood to include the documented processes for identifying hazards associated with a product or service; evaluation and control of those risks; and monitoring of the effectiveness of the risk controls throughout the lifetime of the product or service (e.g. ISO 14971). ${ }^{1}$ This definition applies as much to health care as to other industries.

This article provides an assessment of the risks associated with the insertion and removal of contraceptive implants. The importance of device and equipment design to patient safety in the National Health Service (NHS) has long been recognised in areas such as ambulance design, infection control and drug administration. ${ }^{2}$ Commonly referred to as "mistake-proofing", thoughtful design can help to reduce and manage "use errors". 3 Such errors refer to failures or breakdowns in the use of a device rather than to "human error", which locates the error within the user. In relation to contraceptive implants, three types of incident repeatedly feature in a range of countries: non-insertion, deep insertion and nerve injury during removal. Risks to patient safety relate to the way the insertion device is designed and used, rather than to the pharmacological properties of the implant itself. Suggestions will be made as to how risks identified relating to the use (i.e. insertion and removal) of contraceptive implants may be reduced through intuitive device design and through the management of contributory factors in the environment, within a systematic risk management process.

This article was initially conceived before any information on the redesigned applicator of the new contraceptive implant, Nexplanon ${ }^{\circledR}$, became available. The first part of the article is based on an examination of Implanon ${ }^{\circledR}$, the currently available single-rod implant, and comments on its design following review of the associated risks. The second part is an initial assessment of the design of Nexplanon.

Institute of Clinical Education, Warwick Medical School, University of Warwick, Coventry, UK

Sam Rowlands, LLM, MD, Honorary Associate Professor

Warwick Medical School, University of Warwick, Coventry, UK Mark-Alexander Sujan, PhD, RCUK Academic Fellow

Matthew Cooke, PhD, FCEM, Professor of Emergency Medicine

Correspondence to: Dr Sam Rowlands, Institute of Clinical Education, Warwick Medical School, Gibbet Hill Campus, University of Warwick, Coventry CV4 7AL, UK.

E-mail: sam.rowlands@warwick.ac.uk
Keywords contraceptive implants, design, device, risk management, systems

J Fam Plann Reprod Health Care 2010; 36(4): 191-195 (Accepted 8 September 2010)

\section{Key message points}

- Appreciation of human factors can provide useful insights into the reduction of risks through intuitive device design and the management of contributory factors in the environment.

- Risks of implants relate to the way in which devices are designed and used rather than to their pharmacological properties.

- The risks associated with the use of contraceptive implants include non-insertion and deep insertion.

\section{Background}

Contraceptive implants are highly effective long-acting reversible contraceptives with a good safety record. ${ }^{4}$ Uptake in the UK has been increasing since the publication in 2005 of the NICE guideline on long-acting reversible contraception $^{5}$ and its subsequent promotion nationally. Contraceptive implants are currently used by $1-2 \%$ of women of childbearing age in Britain, ${ }^{6}$ as many as a quarter of a million women. Worldwide, about 6 million women use this method. ${ }^{7}$ With such widespread use, rare adverse effects may nevertheless have a substantial impact on the population.

\section{Breakdown of the insertion procedure into key steps}

Five steps in the insertion procedure provide the basis for analysis of errors that have been reported with Implanon (Box 1). It should be noted that during Steps 1 to 3 , the position of the clinician's hand is constant. The diagram in the Summary of Product Characteristics (SPC) ${ }^{8}$ shows the cannula/needle being held from above between thumb and index finger, by its ridged grip area.

\section{Risks}

\section{Non-insertion}

Non-insertion was not reported with Norplant ${ }^{\circledR}$, the first implant available in the UK. The technique used involved inserting and withdrawing a trocar (six times) but differed

Box 1: Steps in the insertion procedure for Implanon ${ }^{\circledR}$

Step 1 The applicator is picked up and held by the clinician.

Step 2 The skin is punctured with the needle tip.

Step 3 The needle is advanced under the skin.

Step 4 The position of the hands is changed. The seal is broken and the obturator turned through $90^{\circ}$ with one hand, the other hand stabilising the cannula/needle.

Step 5 One hand fixes the obturator while the other hand retracts the cannula/needle. 
from Implanon insertion in that the six Norplant capsules each had to be placed in the trocar by the operator. Implanon is supplied preloaded in its inserter. Noninsertion was reported in the Implanon trials (incidence $0.2 \%$ ) but differed from non-insertions reported from postmarketing surveillance. In the trials, non-insertion was actually recognised at the time of the insertion procedure. 9

Non-insertion has been picked up in post-marketing surveillance in Australia, France and the UK. ${ }^{10}$ The Australian medical indemnity insurer, MDA National, received an unprecedented number of adverse incident reports and responded by applying the Australian and New Zealand Standard for Risk Management. ${ }^{11}$ It was apparent from the Australian analysis that the clinicians were unaware that their technique was faulty. The Royal Australian College of General Practitioners was enlisted to develop guidelines for insertion of Implanon, which included palpation of the implant under the skin after insertion by both the woman and the clinician and documentation of this action.

In retrospect it appears that many clinicians found the procedure of withdrawal of the introducer while keeping the obturator fixed (Step 5) counterintuitive, as it is opposite to the technique used with a syringe and needle. The fact that the placebo implants used in the training sessions were blue and the commercial product was white was found to be confusing, as the implant does not contrast in colour with the obturator. Non-insertion is always due to human error. It was postulated that it could be due to the implant falling out if the needle guard is removed with the needle held at a downward angle. ${ }^{12}$ After this possibility was raised, the manufacturer added the advice that the applicator be held "in the upward position ... until the time of insertion" to the SPC. ${ }^{8}$ However, this potential problem is, in fact, anecdotal: the implant is held tightly in the needle and does not drop out when the applicator is held tip-down and this event has never been documented in published case reports.

Implanon has rigorous manufacture and quality control procedures that accord with Good Manufacturing Practice Guidelines. ${ }^{13}$ The possibility of an implant being absent from the applicator as supplied is very remote. It therefore appears likely that most cases of non-insertion are due to removal of the whole applicator system from the arm with the implant still in situ within the device (error in Step 5). These events generally occur despite clinicians having received appropriate training.

Litigation is rare but has occurred repeatedly in connection with non-insertion of Implanon. ${ }^{10}$ In the experience of the first author, clinicians who are the subjects of errors resulting in litigation are not confined to the untrained, those with limited experience or those who are known to be poor performers.

\section{Deep insertions}

Contraceptive implants should be inserted into the subdermal plane. Deep insertion is generally thought to be associated with poor insertion technique rather than migration of a properly inserted implant. ${ }^{10}$ This problem therefore occurs at Step 3. Insertion instructions have always emphasised the importance of advancing the applicator parallel to the skin surface. Current expert opinion on localisation and removal of deep implants is outlined in the Legal Opinion article by Rowlands in this issue of the Journal (page 243). ${ }^{10}$

\section{Nerve injury}

The recommended site for both Norplant and Implanon was $6-8 \mathrm{~cm}$ above the elbow crease, in the groove between the biceps and triceps muscles. The neurovascular bundle runs quite superficially here; the anatomy is detailed elsewhere. ${ }^{10}$ Although nerve damage is a rare complication of contraceptive implants, it is clearly a serious one. Insertion immediately over the groove is unwise. ${ }^{10}$ In June 2007 the manufacturer revised the SPC, deleting reference to the biceps/triceps groove. The recommended site was changed to $6-8 \mathrm{~cm}$ above the medial epicondyle of the humerus, which is behind the groove. This particular risk is not amenable to solution by improvement of the product design.

\section{Needlestick injury}

To the authors' knowledge this has not been a particular concern with Implanon, but remains a potential risk with the use of any sharp needle.

\section{Discussion}

Scientific writing on implants usually concentrates on the clinical effectiveness and pharmacological safety of the drug itself. ${ }^{14-17}$ However, review of the risks associated with the insertion and removal of contraceptive implants 10 suggests that some of the issues are due to human factors, related to the way clinicians perform the insertion procedure as well as to the conditions under which it is performed. For example, non-insertion and deep insertion are clearly linked to human action and interaction with the device rather than with the pharmacological properties of the drug. Such occurrences are usually referred to as "human error". Could we apply lessons from other industries and other areas of health care to reduce the likelihood of errors in the use of the insertion device (i.e. to "mistake-proof" the activity through design)? Guidance on this question is available. Reason's highly influential accident causation model, 18 the notion of 'Affordances', 19 and Risk Management and Usability Engineering processes as recommended for medical device manufacturers, are all helpful in this regard.

\section{A systems perspective on adverse events}

In safety-critical industries as well as in health care, Reason's accident causation model (the so-called 'SwissCheese' model $)^{18}$ is the standard framework in which to approach and to understand adverse events from a systems perspective. The main assumption of the model is that human errors should be regarded as symptoms of underlying system deficiencies rather than as causes of adverse events as such. Deficient organisational processes include poor processes for specifying and updating procedures, for purchasing and maintaining equipment and stock, for identifying training needs and ensuring access to training, and low priority given to safety and quality. These lead to latent conditions in the work environment where failures are more likely to occur. This is illustrated in Figure 1.

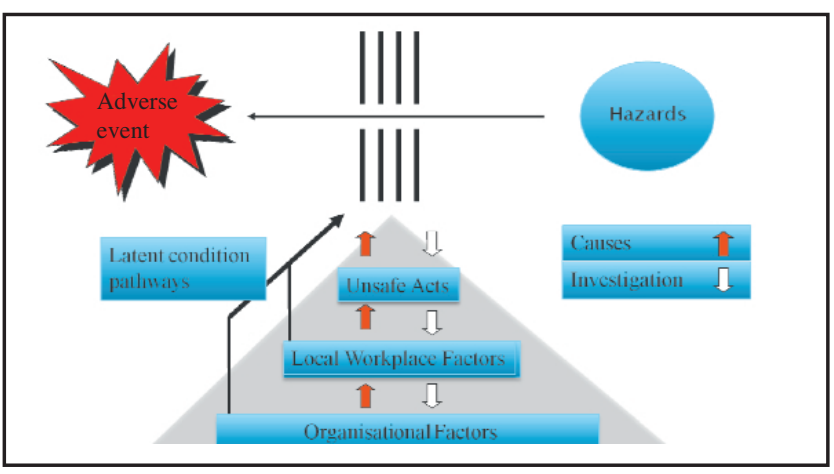

Figure 1 Reason's model of organisational accidents. Figure modified from Managing the Risks of Organizational Accidents. ${ }^{18}$ (c) Ashgate Publishing 
Table 1 Examples of factors affecting the activity of insertion of contraceptive implants and corresponding lead questions for risk management

\begin{tabular}{|c|c|}
\hline Influencing factor & Example of lead questions \\
\hline Task & $\begin{array}{l}\text { - Is the standard procedure for inserting contraceptive implants known to staff? } \\
\text { Do staff have sufficient time available to follow the procedure? } \\
\text { - Are there reported problems with this task? }\end{array}$ \\
\hline Device & $\begin{array}{l}\text { - Has a training package been devised for using the insertion device? } \\
\text { Is the insertion device easy and intuitive to use? } \\
\text { - Are the insertion device and the required materials readily available? }\end{array}$ \\
\hline Individual & $\begin{array}{l}\text { - Is the individual adequately qualified? } \\
\text { Has the individual had access to the training package? } \\
\text { Does the individual have regular opportunity to practise their skills? }\end{array}$ \\
\hline Work environment & $\begin{array}{l}\text { - Is there enough space to carry out this task? } \\
\text { Can the task be carried out free from interruptions? } \\
\text { - Are lighting and noise levels acceptable? }\end{array}$ \\
\hline Organisation & $\begin{array}{l}\text { - Are process measures (e.g. audit or survey) available for this task? } \\
\text { - Are there regular meetings to discuss safety and quality concerns? } \\
\text { - Are communication channels with device manufacturers or regulatory bodies effective? }\end{array}$ \\
\hline
\end{tabular}

This model highlights the importance of giving consideration to the wider context within which contraceptive implants are used. Table 1 provides examples of generic questions that should be addressed within the risk management process. For each influencing factor, examples are given of questions that lead one to explore this particular aspect of risk assessment. The table uses a classification of influencing factors proposed by Vincent $e t$ al..$^{20}$ At present, there is unfortunately little evidence or systematic data available on these factors in relation to the use of contraceptive implants.

\section{Device level: guidance available to manufacturers}

The applicator for insertion of the contraceptive implant is a key element within this activity. Focusing in on the Device level shown in Table 1, what guidance is available to manufacturers? For medical devices guidance exists in the form of international standards that support manufacturers in considering usability aspects and use errors. ISO 14971 (Medical Devices - The Application of Risk Management to Medical Devices $)^{1}$ specifies that manufacturers need to consider reasonably foreseeable misuse (i.e. incorrect or improper use) and have controls in place to reduce the resultant risks to acceptable levels. BS EN 62366 (Medical Devices - Application of Usability Engineering to Medical Devices) ${ }^{21}$ specifies a usability engineering process that allows manufacturers to assess and control risks that result from usability problems associated both with correct use and with use errors.

The common route to controlling risks arising from user interaction with a device is through the provision of training - the intended method of use is reinforced. However, usability engineering also provides principles for the design of medical devices that are intended to make their use as intuitive and safe as possible - mistake- proofing the activity. This is an important factor as devices are being used more widely and by practitioners who may not have received in-depth specialist training 16 or who may not use them regularly. It is also relevant when there is national cascade training and therefore scope for training variation. The discussion below illustrates the types of factor that can be considered and exploited in order to reduce the risk of such interaction failures.

\section{Affordances}

Why do we intuitively know how to hold a pair of scissors? Why can we easily open some doors but struggle with others? The answer to these questions is to be found in those properties of objects that provide clues about their modes of operation. Norman ${ }^{19}$ refers to these perceived and actual properties as affordances. When users take advantage of such affordances, the operation of the device becomes intuitive. Devices that do not exploit the concept of affordances are often more difficult to use and make use errors more likely.

We can apply the concept of affordances to the analysis of the implant applicator used for Implanon to better understand some forms of interaction with the device. It appears that the applicator provides an affordance for a pushing rather than a pulling action, as it looks similar to a syringe. Ideally the applicator should provide an affordance for retraction, as this is the way it is intended to be used. The Implanon applicator could be held with one hand by placing the index finger and thumb as if one were about to give an injection, as indicated by the red arrows in Figure 2. This is particularly relevant as the operator will have given a subcutaneous injection of lidocaine immediately before handling the applicator. This affordance could be perceived to be stronger than the intentionally included affordance for holding the applicator, the ridged grip areas on either side

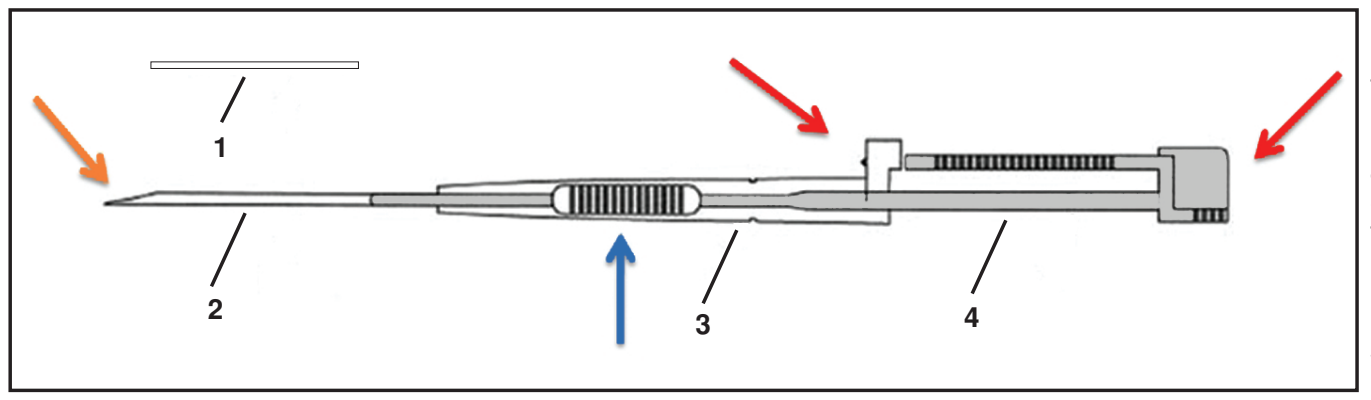

Figure 2 Affordances present in the Implanon ${ }^{\circledR}$ applicator. Key: 1 , Implanon rod; 2, needle; 3, plastic body; 2+3, cannula; 4 , obturator. See text for an explanation of the significance of the coloured arrows. Figure modified from Mascarenhas L, Insertion and removal of Implanon ${ }^{\circledR}$. Contraception 1998; 58(Suppl. 6): 79S-83S. (C) Elsevier, reprinted with permission 
of the cannula, indicated by the blue arrow. This has practical implications for Steps 1 to 3 as described above. The device also provides some visual feedback about the presence or absence of the implant before and after insertion, indicated by the orange arrow.

If the applicator were to be held as if one were about to do a venepuncture, with thumb on top and fingers underneath, then although Step 2 might be accomplished without error, it would be impossible to perform Step 3 in a horizontal direction. The direction of travel of the needle would be angled downwards, resulting in deep insertion.

Holding the applicator incorrectly is also likely to make Step 5 more difficult. The risk here is that the obturator is pushed, rather than fixed. This could then lead to the implant taking the path of least resistance. ${ }^{22}$

Redesign of the implant applicator would ideally eliminate the syringe-likeness that currently overshadows the useful affordance for holding it, and would include an affordance for retraction. As it may not be possible to eliminate the affordance for mistakenly placing one's index finger against the applicator seal, the design could try to make a pushing action meaningless by including a rounded, concave, digit-shaped top for holding the device. The digitshape would indicate where to put one's finger and the rounded edges would prevent the assumption of pushing. Ideally, the design would also include an affordance for pulling the applicator backwards once the needle has been inserted into the skin. In the original applicator design, there is only an affordance for holding it.

\section{Constraints}

Constraints are properties that restrict the ways in which a device can be used. In this particular case, a physical constraint in the form of a forcing function has been designed successfully into the Implanon applicator. A forcing function sets up a pre-condition that has to be met before a particular action can be executed. The safety seal is such a forcing function: it is physically possible to withdraw the cannula/needle only if the safety seal is broken. The design of the device acts as a risk control measure. However, this constraint does not prevent the error of removing the whole applicator from the arm with the implant remaining inside the needle. This error can be made with the seal intact or broken. This is the presumed error that leads to the majority of cases of non-insertion.

\section{Visibility, mapping and feedback}

An intuitive design should make it easy for the user to determine what actions are possible with the device and whether the actions have been carried out successfully or not. The principles of visibility, mapping and feedback play an important role in achieving this. Visibility refers to the property of a device that makes it easy for the user to determine which functions are available, while mapping refers to the property that allows determination of which controls are associated with which functions. Unintuitive or confusing mappings often cause interaction failures. Feedback refers to properties and functions of the device that provide information to the user about what action has been executed and what result has been achieved. Feedback is important in bridging the "gulf of evaluation", 19 that is in increasing the ease with which the user can interpret the state of the device and the extent to which their intentions have been met.

With Implanon the risks related to feedback that are now well recognised are non-insertion and deep insertion. ${ }^{10}$ It is difficult to ascertain whether or not the implant has been successfully deployed. This is principally due to the fact that the implant, once deployed, cannot easily be seen as it is beneath the skin, but there are also issues with the device itself relating to lack of feedback: the device does not provide straightforward indications about whether the implant is contained within the applicator or not. An intuitive design solution should explore ways of providing such feedback. To some extent this has been pursued by ensuring that the implant is visible at the front of the needle. One solution would be for the obturator to be a different colour from the implant. An increase in visibility of the implant at the bevel in the needle tip could further enhance this. Windows or visible areas within the applicator could make it easier to see whether the implant is still present.

\section{Conclusions}

This paper assesses the reported risks associated with the insertion and removal of Implanon, based on 11 years of post-launch clinical experience. In order to investigate the risks further, a formal study consisting of direct observation and analysis of procedures would be needed. Such a study is now unlikely to be commenced as Implanon is about to be replaced with Nexplanon. The documented risks are related to human action rather than to the pharmacological properties of the contraceptive implant. The Discussion reviewed some principles for the design of devices that could reduce such risks and introduced a systems perspective through which adverse events relating to contraceptive implants may be explored. The significant point is that risk management needs to account for the process and the conditions of use as much as for the pharmacological properties. This is particularly true as contraceptive implants are becoming increasingly popular and widespread in use and may be deployed in differing situations and by non-specialists.

It is therefore important to consider human factors at all stages of the development and use of such medical devices. Training of clinicians is obviously essential and is relevant to optimal anatomical placement. However, this is not necessarily a solution to the problems of non-insertion and deep insertion, and is only one of many aspects of risk management that need to be considered. Some recommendations for future development of contraceptive implants are given in Box 2.

\section{An initial assessment of Nexplanon ${ }^{\circledR}$}

Nexplanon, the successor to Implanon, is due for launch in the UK towards the end of 2010. The applicator has been redesigned by the manufacturer and, in addition, the implant itself has been made radio-opaque. ${ }^{23}$ Nexplanon is illustrated in the Commentary by Mansour in this issue of the Journal (page 187). ${ }^{7}$

The Nexplanon applicator has the following new features when compared to the Implanon applicator: 23

- The entire shape and mechanism are different.

- The applicator is rendered unusable if no implant is present within the needle. In this situation the needle protection cap cannot be removed.

- The applicator sets a predetermined depth for insertion of the implant beneath the skin surface.

Box 2: Recommendations for future development of contraceptive implants

- Collect systematic data about the incidence, nature and type of risks associated with the use of implants.

- Establish the extent to which the various influencing factors contribute to those risks in practice.

- Incorporate human factors principles into risk management.

- Exploit affordances, visibility and feedback principles in the design of applicators to make use errors less likely. 
- The finger-operated slider on the top surface facilitates one-handed completion of the insertion procedure. This addresses difficulties with Implanon insertion Steps 4 and 5 .

- After completion of the backward movement of the slider, the needle is completely inside the body of the applicator, so reducing the risk of needlestick injury.

- The contrasting colour of the obturator improves differentiation from the implant.

The manufacturer recommends that the operator should perform the insertion procedure while seated, so that some degree of direct visualisation of the needle is possible.

It appears that the concept of affordances has been used in the redesign. The affordance for holding during introduction of the needle into the skin remains, with some modification. The syringe-likeness of the applicator has been changed. There is now a slider on top of the device with an indented shape that invites placement of a finger onto it. This is remarkably like the suggestion made above by the authors of this article. The one-handed release of the implant avoids the coordination of the hands previously needed in Steps 4 and 5. The constraint of unlocking the safety seal has been removed in the redesign. This seal was specific to the old design and is not needed in the new applicator.

The use of constraints is also evident. Setting the depth to which the needle can be inserted beneath the skin surface makes it more difficult to carry out a deep insertion. Also, making the applicator unusable if no implant is present within the needle is a forcing function, setting up a precondition that the implant be present before the action can continue.

The obturator and implant are now in different colours. This is an example of the principle of visibility. However, visualisation of the implant by the operator during the procedure is reduced compared to Implanon. Feedback as to whether or not the implant has been successfully deployed is therefore limited. This may well be the reason behind some problems already encountered with use of Nexplanon. ${ }^{23}$ Visualisation of the needle during insertion is restricted because the 'nose' of the applicator obscures the view. This does not seem to be fully overcome by conducting the insertion in the sitting position, viewing the procedure from the side. Clinicians have reported that there is a need for better visualisation of the needle during insertion. In addition, a new problem has emerged, namely partial expulsion of the implant. This is possibly related to the operator failing to insert the needle to its full length, or to the device being allowed to move backward when pulling back on the slider. $^{23}$

With the launch of the new product, manufacturers, risk managers and clinicians should continue to review the safety of the method, particularly bearing in mind human factors in relation to the use of the applicator. It is hoped that the redesign will reduce the risks of noninsertion, deep insertion and needlestick injury. However, the redesign cannot reduce the risks associated with operator placement of the implant in inappropriate anatomical sites. Further assessment of problems such as partial expulsion, and of any other unforeseen issues, will only be possible after Nexplanon has come into general clinical use.
Statements on funding and competing interests Funding None identified.

Competing interests The first author has received payment for delivering training sessions for both Norplant ${ }^{\circledR}$ and Implanon ${ }^{\circledR}$ and has had expenses paid for attending scientific congresses by companies marketing these implants.

\section{Editor's note}

Interested readers should also see Diana Mansour's Commentary article on Nexplanon ${ }^{\circledR}$ (page 187) and Sam Rowlands' Legal Opinion article about contraceptive implants (page 243) of this issue of the Journal.

\section{References}

1 International Organization for Standardization. ISO 14971 Medical Devices - Application of Risk Management to Medical Devices. Geneva, Switzerland: International Organization for Standardization, 2007.

2 Tonks A. Patient safety: safer by design. BMJ 2008; 336: 186-188.

3 Grout J, College B, Rome GA. Mistake-proofing the Design of Health Care Processes. AHRQ publication 07-0020. Rockville, MD: Agency for Healthcare Research and Quality, 2007.

4 Darney P. Implantable contraception. Eur J Contracept Reprod Health Care 2000; 5(Suppl. 2): 2-11.

5 National Collaborating Centre for Women's and Children's Health. Long-acting Reversible Contraception (NICE Clinical Guideline CG30). 205. http://www.nice.org.uk/CG030 [Accessed 6 September 2010].

6 Lader D. Contraception and Sexual Health, 2008/09. Newport, UK: Information Centre, 2009.

7 Mansour D. Nexplanon ${ }^{\circledR}$ : what Implanon ${ }^{\circledR}$ did next. J Fam Plann Reprod Health Care 2010; 36: 187-189.

8 Organon Laboratories Ltd. Implanon: 68mg implant for contraceptive use: Summary of Product Characteristics. 2009. http://www.medicines.org.uk/EMC/medicine/5382/SPC/ Implanon+68mg+implant+for+subdermal+use/ [Accessed 6 September 2010].

9 Mascarenhas L. Insertion and removal of Implanon ${ }^{\circledR}$. Contraception 1998; 58(Suppl. 6):79S-83S.

10 Rowlands S. Legal aspects of contraceptive implants. J Fam Plann Reprod Health Care 2010; 36: 243-248.

11 Wenck BCA, Johnston PJ. Implanon and medical indemnity: a case study of risk management using the Australian standard. Med J Aust 2004; 181: 117-119.

12 Mansour D, Fraser IS, Walling M, Glenn D, Graesslin O, Egarter $\mathrm{C}$, et al. Methods of accurate localisation of nonpalpable subdermal contraceptive implants. J Fam Plann Reprod Health Care 2008; 34: 9-12.

13 European Commission. Good Manufacturing Practice Guidelines (EuraLex Vol. 4). Brussels, Belgium: European Commission, 2009.

14 Edwards JE, Moore A. Implanon ${ }^{\circledR}$ : a review of clinical studies. Br J Fam Plann 1999; 24(Suppl.): 3-16.

15 Newton J, Newton P. Implanon ${ }^{\circledR}$ - the single-rod subdermal contraceptive implant. J Drug Eval 2003; 1: 181-218.

16 Meirik O, Fraser IS, d'Arcangues C. Implantable contraceptives for women. Hum Reprod Update 2003; 9: 49-59.

17 Darney P, Patel A, Rosen K, Shapiro LS, Kaunitz AM. Safety and efficacy of a single-rod etonogestrel implant (Implanon): results from 11 international clinical trials. Fertil Steril 2009; 91: 1646-1653.

18 Reason J. Managing the Risks of Organizational Accidents. London, UK: Ashgate Publishing, 1997.

19 Norman DA. The Psychology of Everyday Things. New York, NY: Basic Books, 1987.

20 Vincent C, Taylor-Adams S, Stanhope N. Framework for analysing risk and safety in clinical medicine. BMJ 1998; 316: $1154-1157$.

21 British Standards Institution. BS EN 62366. Medical Devices Application of Usability Engineering to Medical Devices. Bristol, UK: British Standards Institution, 2008.

22 Walling M. How to remove impalpable Implanon ${ }^{\circledR}$ implants. J Fam Plann Reprod Health Care 2005; 31: 320-321.

23 Mansour D, Mommers E, Teede H, Sollie-Eriksen B, Graesslin $\mathrm{O}$, Ahrendt $\mathrm{HJ}$, et al. Clinician satisfaction and insertion characteristics of a new applicator to insert radiopaque Implanon: an open-label, noncontrolled, multicenter trial. Contraception 2010; 82: 243-249.

Readers are advised that due to the ongoing transition to the Journal's new commercial publisher, BMJ Group, it is likely that dispatch of Faculty Members' personal copies of the January 2011 issue will take place a week or two later in January 2011 than in previous years. The January 2011 issue is, however, scheduled for online publication at the end of December 2010, and details of how to access this issue online will be available on the Faculty website (www.fsrh.org). 